\section{Tag des Prostatakarzinoms am 14. September 2000}

Das Prostatakarzinom ist das am häufigsten diagnostizierte Karzinom beim älteren Mann und steht an zweiter Stelle hinter dem Bronchuskarzinom bezüglich Krebssterblichkeit. Eine steigende Inzidenz, zusammen mit einer aufgrund der zunehmenden Lebenserwartung sich vergrössernden Risikopopulation, machen das Prostatakarzinom zu einem gewichtigen gesundheitspolitischen und sozio-ökonomischen Problem.

Unsere Diagnose- und Behandlungsstrategien müssen im Lichte des sich ausbreitenden Kostenbewusstseins einer sensibilisierten Ärzteschaft, der Versicherer unter dem Druck der Sozialversicherungen und, nicht zuletzt, der Patienten, welche zunehmend kritischer und informierter, aber auch verunsicherter sind, durchdacht und hinterfragt werden. Viele offene - und oft praktische - Fragen bedürfen einer dringenden Beantwortung: Ist es sinnvoll, bei einem beschwerdefreien 80jährigen Patienten eine PSA-Bestimmung durchzuführen? Ist ein generelles Prostatakarzinomscreening der männlichen Bevölkerung angebracht? Welchen Stellenwert haben die verschiedenen therapeutischen Möglichkeiten - und wann und bei wem sind sie angezeigt? In welche Richtung zeigen die neueren Trends in der Behandlung des Prostatakarzinoms? Gibt es diätetische oder andere präventive Ansätze?

Aufgrund dieser Problematik hat die Schweizerische Krebsliga (SKL) das Prostatakarzinom zum Schwerpunkt ihrer Anstrengungen im Jahre 2000 gemacht. Unter dem Patronat und im Auftrag der Verbindung der Schweizer Ärztinnen und Ärzte FMH und der SKL hat die Schweizerische Gesellschaft für Urologie am 14. September 2000 einen Fortbildungstag rund um das Thema Prostatakarzinom für Ärzte in der Praxis organisiert. Gerade weil den Hausärzten eine zentrale Rolle bei der Führung der Patienten und der diagnostischen und therapeutischen Weichenstellung zukommt, haben die schweizerischen Urologen mit der Unterstützung der Schweizerischen Gesellschaften für Allgemeine (SGAM) und Innere (SGIM) Medizin diesen Fortbildungstag zum Bestandteil ihres Jahreskongresses gemacht.

Wir möchten daher alle praktisch tätigen und interessierten Kollegen herzlich zu dieser Fortbildungsveranstaltung am 14. September 2000 in Interlaken einladen.

Die Programmverantwortlichen: G. Thalmann, U. E. Studer Urologische Universitätsklinik Bern 